\title{
A randomized controlled trial comparing nociception level (NOL) index, blood pressure, and heart rate responses to direct laryngoscopy versus videolaryngoscopy for intubation: the NOLint project
}

\section{Une étude randomisée contrôlée comparant les réponses de l'indice de niveau de nociception (NOL), la pression artérielle et la fréquence cardiaque à une laryngoscopie directe versus vidéolaryngoscopie pour intubation: le projet NOLINT}

\begin{abstract}
Virginie Sbeghen, MD, FRCPC · Olivier Verdonck, MD, MSc, DESAR · Jason McDevitt, MD, MSc, FRCPC • Valérie Zaphiratos, MD, MSc, FRCPC ・ Véronique Brulotte, MD, MSc, FRCPC · Christian Loubert, MD, FRCPC · Issam Tanoubi, MD, MA(ed), DESAR · Pierre Drolet, MD, FRCPC · Marie-Eve Belanger, MD, PGdip(ed), FRCPC • Louis-Philippe Fortier, MD, MSc, FRCPC • Nadia Godin, RN • Marie-Claude Guertin, PhD • Annik Fortier, MSc $\cdot$ Philippe Richebé, MD, PhD, DESAR 10
\end{abstract}

Received: 2 May 2020/Revised: 26 November 2020/Accepted: 1 December 2020/Published online: 11 March 2021

(C) Canadian Anesthesiologists' Society 2021

\begin{abstract}
Purpose The effect of direct laryngoscopy using a Macintosh blade (MAC) vs GlideScope ${ }^{T M}$ videolaryngoscopy using a Spectrum LoPro blade (GVL) on nociceptive stimulation has not been quantitatively studied. This study used the new nociception level (NOL) index to compare the nociceptive response induced by GVL or MAC during laryngoscopy with or without intubation.
\end{abstract}

Supplementary Information The online version of this article (https://doi.org/10.1007/s12630-021-01936-0) contains supplementary material, which is available to authorized users.

V. Sbeghen, MD, FRCPC · O. Verdonck, MD, MSc, DESAR J. McDevitt, MD, MSc, FRCPC · V.Zaphiratos, MD, MSc, FRCPC

V. Brulotte, MD, MSc, FRCPC · ChristianLoubert, MD, FRCPC I. Tanoubi, MD, MA(ed), DESAR · P. Drolet, MD, FRCPC . M.-E. Belanger, MD, PGdip(ed), FRCPC · L.-P. Fortier, MD, MSc, FRCPC · N. Godin, RN · P. Richebé, MD, PhD, DESAR ( $\triangle)$ Department of Anesthesiology and Pain Medicine of University of Montreal, and the Department of Anesthesiology and Pain Medicine of Maisonneuve Rosemont Hospital, CIUSSS de l'Est de l'Ile de Montréal, University of Montreal, 5415, Boulevard de l'Assomption, Montréal, QC H1T 2M4, Canada

e-mail: philippe.richebe@umontreal.ca

M.-C. Guertin, $\mathrm{PhD}$ - A. Fortier, MSc

Department of Statistics, Montreal Health Innovations

Coordinating Center (MHICC), Montréal, QC, Canada
Methods Patients underwent two laryngoscopies at fourminute intervals $(L 1, L 2)$, one with GVL and the other with MAC (first randomization). A third laryngoscopy (L3) followed by tracheal intubation was performed four minutes after L2 (GVL or MAC, second randomization). Nociception was quantitatively assessed by NOL and standard hemodynamic parameters (heart rate [HR] and mean arterial pressure). For the crossover design, blade comparisons accounted for sequence and blade type. A possible carryover effect between laryngoscopies was assessed.

Results In the 50 patients randomized, there was no carryover effect from one laryngoscopy to the next for all analyzed parameters. Nociception level index peak values were higher with MAC than GVL. Analysis of $\triangle N O L$ showed a lower nociceptive response with $G V L$ for $L 1+L 2$ combined. Mean peak NOL values were significantly higher after L3+intubation than after $L 1+L 2$, for both GVL and MAC groups. Analysis of $\triangle H R$ values did not show a significant difference between GVL and MAC for any laryngoscopy.

Conclusion Laryngoscopy alone with GVL induces less nociception than with MAC. The NOL was more sensitive than $H R$ at detecting nociceptive responses to MAC vs GVL. Additionally, and irrespective of which techniquel blade was used, the combination of laryngoscopy + 
tracheal intubation produced a much greater nociceptive response than the laryngoscopy alone.

Trial registration www.clinicaltrials.gov (NCT03277872); registered 29 August 2017.

\section{Résumé}

Objectif L'effet de la laryngoscopie directe avec une lame Macintosh (MAC) par rapport à la vidéolaryngoscopie à l'aide d'un GlideScope ${ }^{T M}$ avec lame Spectrum LoPro (GVL) sur la stimulation nociceptive n'a pas été quantitativement étudié. Cette étude a utilisé le nouvel indice de niveau de nociception (NOL) pour comparer la réponse nociceptive induite par une laryngoscopie avec GVL ou MAC avec ou sans intubation.

Méthode Les patients ont subi deux laryngoscopies à des intervalles de quatre minutes (L1, L2), l'une par GVL et l'autre par MAC (première randomisation). Une troisième laryngoscopie (L3) suivie d'une intubation trachéale a été effectuée quatre minutes après L2 (GVL ou MAC, deuxième randomisation). La nociception a été quantitativement évaluée à l'aide de l'indice NOL, et les paramètres hémodynamiques standard (fréquence cardiaque [FC] et pression artérielle moyenne) ont été mesurés. Dans le volet croisé de l'étude, les comparaisons de lames ont tenu compte de la séquence et du type de lame. La possibilité d'un effet de persistance entre les laryngoscopies a été évaluée.

Résultats Chez les 50 patients randomisés, il n'y a eu aucun effet de persistance d'une laryngoscopie à la suivante pour tous les paramètres analysés. Les valeurs maximales de l'indice de nociception étaient plus élevées avec les lames MAC qu'avec la vidéolaryngoscopie GVL. L'analyse de $\triangle N O L$ a montré une réponse nociceptive inférieure avec la vidéolaryngoscopie GVL pour $L 1+L 2$ combinés. Les valeurs maximales moyennes de NOL étaient significativement plus élevées après L3+intubation qu'après $L 1+L 2$, tant pour les groupes GVL que MAC. L'analyse des valeurs $\triangle F C$ n'a pas montré de différence significative entre les techniques GVL et MAC pour quelque laryngoscopie que ce soit.

Conclusion La laryngoscopie seule avec le GlideScope induit moins de nociception qu'avec une lame MAC. L'indice NOL était plus sensible que les FC pour détecter les réponses nociceptives à la laryngoscopie MAC vs GVL. En outre, et indépendamment de la technique/lame utilisée, la combinaison de laryngoscopie + intubation trachéale a produit une réponse nociceptive beaucoup plus importante que la laryngoscopie seule.

Enregistrement de l'étude www.clinicaltrials.gov (NCT03277872); enregistrée le 29 août 2017.
Keywords GlideScope ${ }^{\mathrm{TM}}$ Spectrum $^{\mathrm{TM}}$ angulated LoPro Single-Use Blade $\cdot$ intubation · laryngoscopy · Macintosh blade $\cdot$ nociception $\cdot$ NOL index

The advent and increasing availability of videolaryngoscopy as an alternative to direct laryngoscopy for endotracheal intubation has elicited a number of studies comparing these two techniques. Recent studies have explored a variety of parameters, including success of intubation, ${ }^{1-5}$ the relative force required to perform the laryngoscopy, ${ }^{4,6-11}$ and the hemodynamic responses associated with video vs direct laryngoscopy. ${ }^{12,13}$

GlideScope $^{\mathrm{TM}}$ videolaryngoscopy (GVL) with a Spectrum $^{\mathrm{TM}}$ hyperangulated LoPro single use blade (Verathon, Inc. Bothell, WA, USA) has a number of potentially interesting attributes as an alternative to direct laryngoscopy using a traditional Macintosh blade (MAC). For example, GVL provides better glottic exposure and has a better intubation success rate than MAC. ${ }^{5,14}$ Moreover, GVL was found to be $99 \%$ successful for intubation after initial failure of MAC. ${ }^{3}$ The sharp angulation of the classic blade used during GVL reduces cervical spine motion by up to $50 \%$ compared with the blade used for MAC. ${ }^{5}$ Additionally, the use of GVL may also diminish the risk of soft tissue lacerations. ${ }^{7,11}$ Given that the data suggest less force is exerted on tissue when using GVL, a reasonable hypothesis might be that it is relatively less stimulating than traditional MAC.

Anesthesiologists have often used vital signs such as heart rate (HR) and blood pressure as indicators of intraoperative nociception. Nevertheless, existing studies comparing the hemodynamic responses of direct laryngoscopy with videolaryngoscopy have not consistently shown a difference between the two. ${ }^{12,13}$ There is, of course, an inherent limitation of such studies, namely that HR and blood pressure can vary for reasons other than nociception.

A device or index to directly measure pain and/or nociception would obviate such limitations. The PMD200 ${ }^{\mathrm{TM}}$ (Medasense Biometrics Ltd., Ramat Gan, Israel) device is designed to do so by offering an dimension-less index called the nociception level (NOL) index. ${ }^{15-19}$ This index varies from 0 to 100 (100 represents the maximum level of nociception and 0 represents total absence of nociception). The NOL index is generated from a non-linear Random Forest regression using five different parameters gathered from a finger probe connected to the PMD200 ${ }^{\mathrm{TM}}$. These parameters are HR, HR variability at the $0.15-$ to $0.4-\mathrm{Hz}$ band power, amplitude of the 
photoplethysmogram, skin conductance level, number of skin conductance fluctuations, and their time derivatives.

Previous research on the PMD200 ${ }^{\mathrm{TM}}$ device and its NOL index found that a threshold of 25 might be considered an appropriate threshold for nociception detection. ${ }^{15-19}$ The NOL index was shown to correlate well with noxious stimuli of different intensities. ${ }^{16-19}$ It also performed better than standard hemodynamic parameters, such as HR and blood pressure, for the evaluation of nociception under general anesthesia. ${ }^{16-19}$

The present study aimed to compare GVL with MAC in terms of NOL index, HR, mean arterial blood pressure (MAP) and bispectral index (BIS ${ }^{\mathrm{TM}}$; Medtronic, Minneapolis, MN, USA) responses measured before, during, and immediately following laryngoscopy and/or laryngoscopy + intubation. We hypothesized that NOL index values would rise higher after a MAC than with GVL.

\section{Methods}

This trial was conducted at the Maisonneuve-Rosemont University Hospital, CIUSSS de l'Est de l'Ile de Montreal (university-affiliated teaching hospital performing more than 17,000 surgeries each year, with 800 beds), University of Montreal, Montreal, QC, Canada, between September and November 2017. The study was approved by the local scientific and ethic committee (Comité d'Ethique de la Recherche, installation Hôpital Maisonneuve-Rosemont, CIUSS de l'Est-de-l'île-de-Montréal, Montréal, QC, Canada; approval number 2018-1144) and was registered on clinicaltrials.gov (NCT03277872). All patients signed an informed consent form and all electronic data were stored anonymously.

\section{Patient population—inclusion/exclusion criteria}

The inclusion criteria were: 1) American Society of Anesthesiologists physical status I or II, 2) Mallampati class 1 or 2,3) > $18 \mathrm{yr}$ old, and 4) having elective general, gynecological, neurologic, orthopedic, plastic, or urological surgery under general anesthesia requiring endotracheal intubation. The exclusion criteria were: 1) refusal to participate, 2) history of psychiatric diseases or psychological problems (including developmental delay), 3) inability to give consent, e.g., because of language barrier, 4) anticipated difficult airway (Mallampati class 3 and 4 , thyromental distance $<6 \mathrm{~cm}$, mouth opening $<3$ $\mathrm{cm}$, neck extension $<80^{\circ}$ and neck flexion $<35^{\circ}$, or inability to protrude the mandible adequately as assessed by upper lip bite test), 5) history of neck rigidity or instability, 6) body mass index $>35,7$ ) presence of a beard (because of recognized risk of difficult ventilation), 8) history of oropharyngeal or tracheal surgery (excluding adenoidectomy, amygdalectomy, or tooth removal), 9) severe coronary artery disease, 10) serious cardiac arrhythmias (including atrial fibrillation), 11) use of $\beta$ blockers, 12) history of opioid or illicit drug use, 13) allergy to remifentanil or propofol, 14) pregnancy, and 15) contraindications to mask ventilation (gastrointestinal tract obstruction, pregnancy, active gastroesophageal reflux disease, non-fasting patients).

Patients with unexpected difficult airway (CormackLehane grade 3 or 4) and patients requiring hemodynamic support with vasopressors or inotropes at any time from five minutes preceding the first laryngoscopy to five minutes after the insertion of endotracheal tube (because of possible interaction with NOL interpretation) were excluded from the study. All adverse events (such as hypo/ hypertension, brady/tachycardia, etc.) were recorded and patients who required treatments for such events were excluded from study. See Fig. 1A for the study flowchart.

Study design

This study was a prospective, randomized-controlled, single-centre superiority trial with two parallel groups (1:1 allocation) (Fig. 1). Two laryngoscopy groups were defined on the basis of technique and device - the GVL group underwent GlideScope ${ }^{\mathrm{TM}}$ videolaryngoscopy with a Spectrum $^{\mathrm{TM}}$ angulated LoPro single-use blade, and the MAC group underwent direct laryngoscopy with a Macintosh reusable blade. To isolate the nociceptive response associated with the laryngoscopy (i.e., to subtract out the stimulation of the insertion of an endotracheal tube), each participant first underwent laryngoscopy (no insertion of the endotracheal tube) with both the GVL and MAC techniques. A first randomization (randomization \#1; with 1:1 allocation) determined the order of the two "isolated" laryngoscopies. This aspect of the study added a crossover design to control for any effects related to the order of the laryngoscopy techniques. Participants were then randomized again (randomization \#2) into one of the two following groups: 1) tracheal intubation after GVL, or 2) tracheal intubation after MAC. Allocation to these groups were 1:1 with 25 participants in each group (Fig. 1). The NOL index, hemodynamic parameters (HR and MAP), and BIS index were recorded for all laryngoscopies, including the final laryngoscopy + tracheal intubation.

Randomization was achieved using an electronic randomization list generated by an independent research assistant and the code was kept in opaque sequentially numbered envelopes. Each envelope contained the result for the two randomizations. The operator and the research 


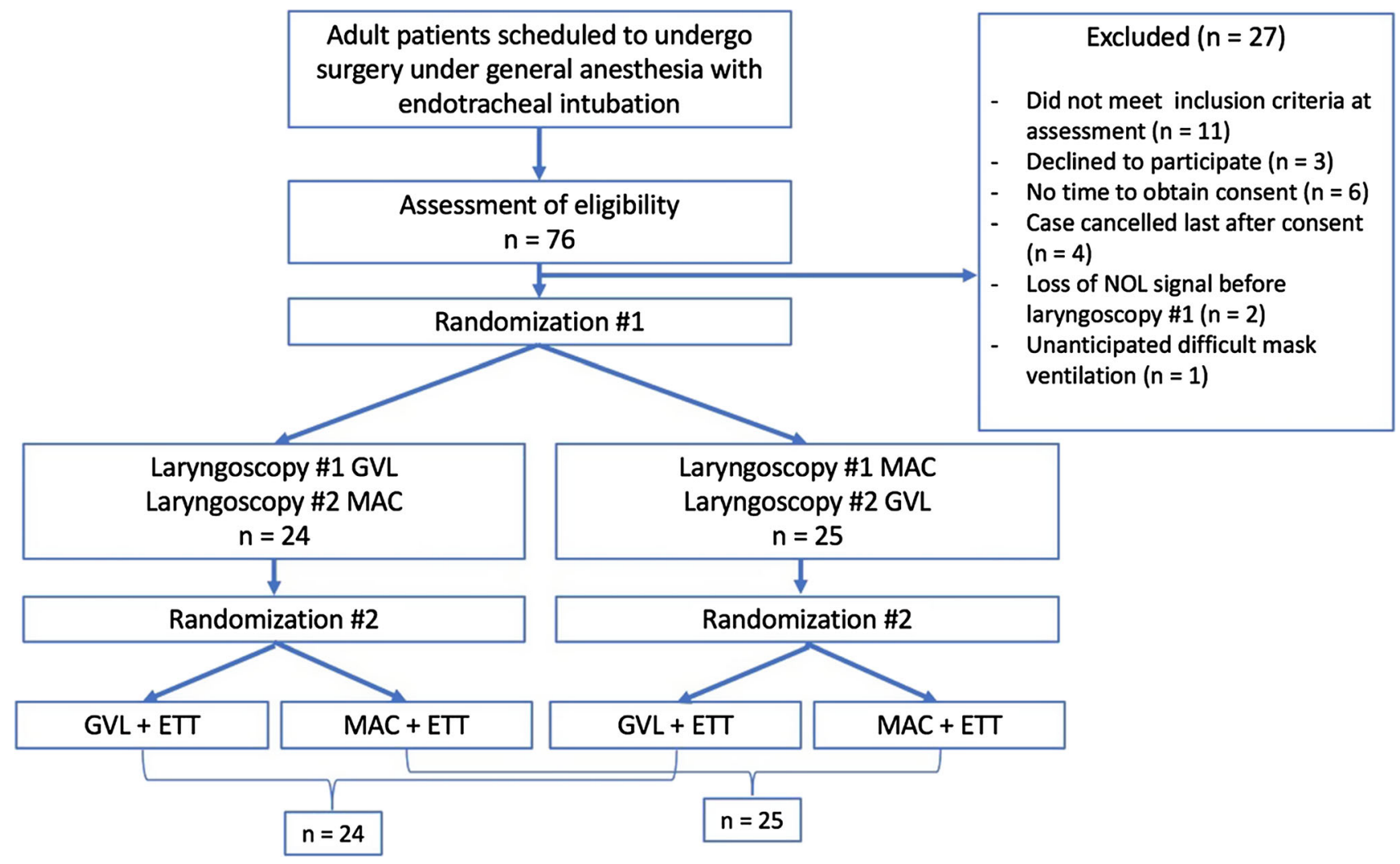

Fig. 1 Flowchart of the study; crossover study design with first randomization for laryngoscopies \#1 and \#2, then second randomization for laryngoscopy \#3 + tracheal intubation.

team were not blinded to the treatment assignment; however, all the parameters assessed during the 18-min study period were non-operator dependent criteria, thus limiting the risk of bias due to the lack of blinding. Additionally, all laryngoscopies were performed by only two experienced anesthesiologists to limit the risk of nociception variation induced by different operators.

Interventions/conduct of the study

After the patient's arrival in the operating room, intravenous midazolam $\left(0.02 \mathrm{mg} \cdot \mathrm{kg}^{-1}\right)$ was administered. Preoxygenation (with $100 \% \mathrm{O}_{2}$ ) was started to reach an expired $\mathrm{O}_{2}$ concentration over $90 \%$. While the patient was preoxygenated, three measures (blood pressure, HR, and NOL index) were recorded with one-minute interval between each to establish a baseline. All data were electronically registered every five seconds for the entire duration of the study for each patient (except for blood pressure, which was evaluated every minute for the duration of the study).

General anesthesia was first induced by introducing lidocaine $\left(1 \mathrm{mg} \cdot \mathrm{kg}^{-1}\right)$ intravenously. Then an intravenous propofol bolus $\left(2 \mathrm{mg} \cdot \mathrm{kg}^{-1}\right)$ followed by an infusion (100 $\left.\mu \mathrm{g} \cdot \mathrm{kg}^{-1} \cdot \mathrm{min}^{-1}\right)$ via an infusion pump and subsequently titrated for a BIS index value between 45 and 55 . Simultaneously, remifentanil was administered intravenously $\left(1.0 \mu \mathrm{g} \cdot \mathrm{kg}^{-1}\right.$ bolus given over $30 \mathrm{sec}$, followed by an infusion of $\left.0.05 \mu \mathrm{g} \cdot \mathrm{kg}^{-1} \cdot \mathrm{min}^{-1}\right)$. The end of the remifentanil bolus was considered $\mathrm{T} 0$ of the study. Once loss of consciousness occurred and the patient became apneic, a Guedel Airway $\mathrm{DAR}^{\mathrm{TM}}$ (Covidien, Mansfield, MA, USA) oral airway device was inserted to facilitate manual ventilation until the first laryngoscopy was performed (size chosen according to manufacturer specifications based on patient weight and height). The patient was manually ventilated with a face mask. If the manual ventilation was easy, rocuronium $\left(0.8 \mathrm{mg} \cdot \mathrm{kg}^{-1}\right)$ was injected. All drugs were given according to the patient's adjusted body weight (ABW). Adjusted body weight was calculated with the Robinson's formula: $A B W=I B W+$ $[0.4 \times(T B W-I B W)]$ where $T B W$ is total body weight and $I B W$ is ideal body weight. ${ }^{20-23}$

At T5 (i.e., five minutes after T0), the first laryngoscopy was performed (either using GVL or MAC techniques, according to the first randomization). The size of the disposable blade for GVL and reusable blade for MAC was decided according to the patient's characteristics (height and weight). Patients were positioned supine with neck in extension (classical "sniff-position pillow" position). The 


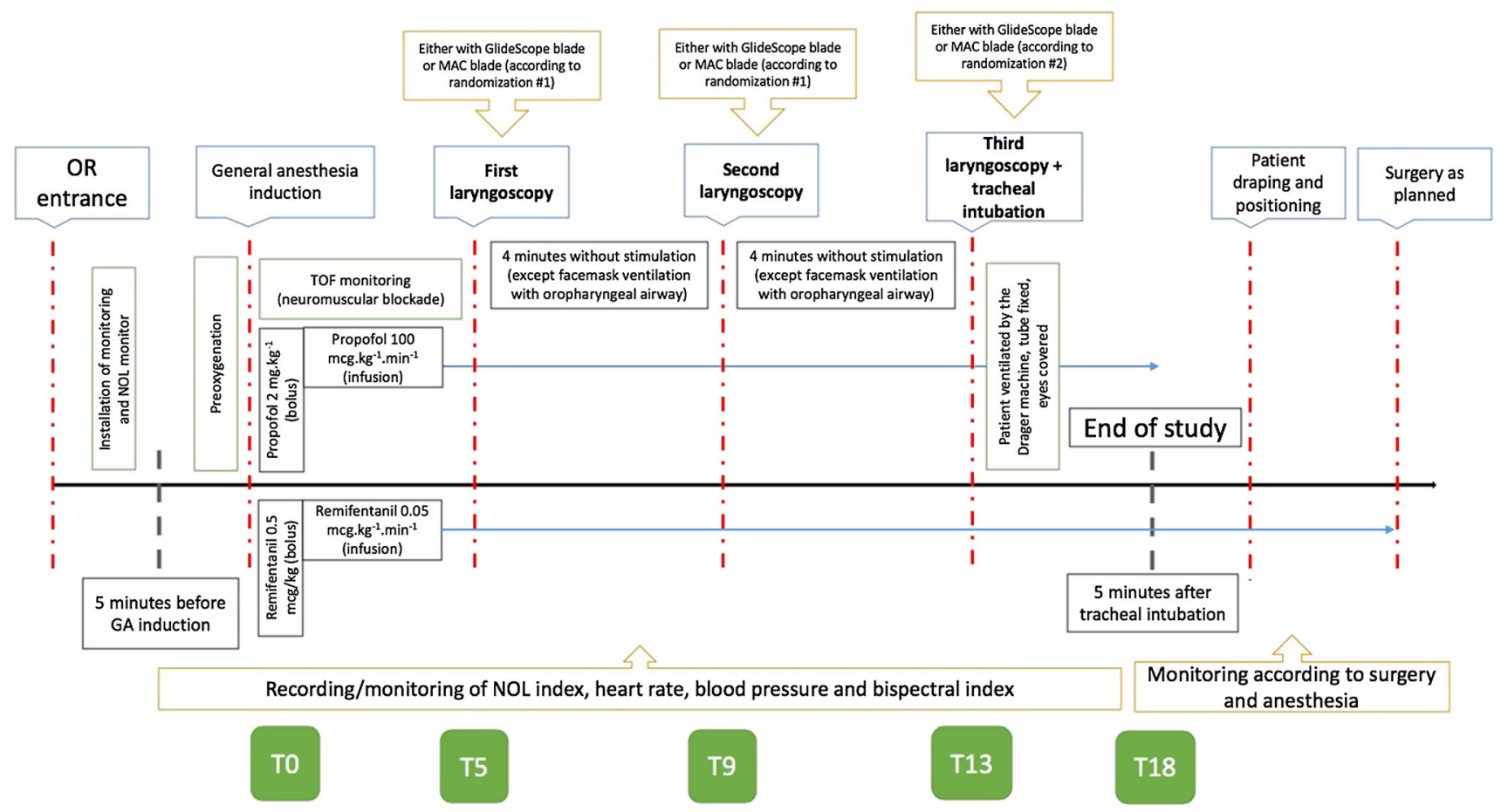

Fig. 2 Study design and timeline of all the study procedures.

laryngoscopy was performed to obtain the best CormackLehane grade possible for each patient (grade 1 or 2). If any laryngoscopy attempt (MAC or GVL) took more than $30 \mathrm{sec}$ to perform, and if no grade 1 or 2 view was obtained within $30 \mathrm{sec}$, the laryngoscopy was considered difficult and the patient was excluded from the analysis.

Following this first laryngoscopy, the operator replaced the oropharyngeal airway device and ventilated the patient with a face mask $\left(100 \% \mathrm{O}_{2}\right)$ for four minutes without stimulating the patient otherwise.

At T9 (nine minutes after T0; four minutes after laryngoscopy \#1), the second laryngoscopy was performed (with MAC if the first laryngoscopy was GVL, or vice-versa). Again, only laryngoscopy was performed, and the endotracheal tube was not inserted. Following this second laryngoscopy, the operator inserted the oropharyngeal airway device and ventilated the patient with a face mask $\left(100 \% \mathrm{O}_{2}\right)$ for four additional minutes, without otherwise stimulating the patient.

At T13 (13 min after T0), the third laryngoscopy was performed (either with GVL or MAC techniques, according to the second randomization). This time, the endotracheal tube was inserted (size 7 or 8 , depending on the size and sex of the patient). Once inserted, the tube was secured, and the patient's eyes were protected as per standard anesthetic practice. The patient was then ventilated with the anesthesia machine (Drager Perseus A500; Drager Medical Canada Inc, Mississauga, ON, Canada) but without any other stimulation for the five subsequent minutes while all the study parameters continued to be recorded. At T18, the study ended. The anesthesia then continued at the discretion of the anesthesiologist in charge of the operating room. See Fig. 2 for study design with all interventions and assessment according to SPIRIT guidelines.

All laryngoscopies/intubations were performed by only two experienced anesthesiologists to minimize variability arising from multiple and/or less experienced operators.

Data collection and handling

All the intraoperative data were recorded with an automated information management system. The PMD$200^{\mathrm{TM}}$ monitor device recorded intraoperative NOL values every five seconds. Other intraoperative parameters (HR, MAP, BIS, etc.) were automatically and electronically collected every five seconds from the Perseus ${ }^{\circledR}$ A500 anesthesia workstation combined to the physiologic monitor M540 (Dräger AG \& Co., Lübeck, Germany). At the end of the procedure, all data were anonymously transferred and stored into a protected computer data file for further statistical analysis.

All data on the PMD200 ${ }^{\mathrm{TM}}$ monitor were electronically collected every five seconds. The BIS, HR, and MAP were also electronically recorded every five seconds from the anesthesia monitors. Peak values of each parameter after nociceptive stimulations were identified manually and confirmed using the automatic peak function of GraphPad 
Prism for each parameter (GraphPad Prism version 7.03 for Windows, GraphPad Software, La Jolla, CA, USA; www. graphpad.com). Then, data from the peak value \pm ten seconds (two data points before the peak, the peak, then two data points after the peak) were averaged (five data points total) to avoid a peak due to any artefact for each of the recorded parameter. Absolute differences ("delta" for each parameter) between baseline values (pre-stimulation) and post-stimulation values were calculated using an average of the last $30 \mathrm{sec}$ of the baseline (before the stimulation) and the average of the five data points representing the peak post-stimulation for each parameter. Area under the curve (AUC) was calculated with the trapezoidal method using values collected from $\mathrm{T} 0$ until $\mathrm{T}+3$ min after the laryngoscopy, for each study parameter.

\section{Sample size calculation}

To meet the study objectives, which were to compare MAC vs GVL in parallel and/or crossover design, a sample size based on a $t$ test was used (parallel design). The resulting sample size per group would assure a sufficient number of subjects for a group comparison on paired data (crossover design). A previous study of 30 patients in our centre using a similar protocol for general anesthesia showed a mean increase in NOL index values after direct laryngoscopy with the Macintosh laryngoscope of 17 with a standard deviation (SD) of 7. Also, previous research suggests the magnitude of the key indicators of hemodynamic response (e.g., MAP) increase by approximately 20-30\% from baseline values shortly after intubation in anesthetized individuals having received conventional doses of narcotics. $^{13}$ Based on these results, we expected a reduction of the nociceptive response to laryngoscopy by about 35\% when using the GVL technique (going from 17 \pm 7 to $11 \pm 7$ in terms of NOL index variation after laryngoscopy). With $\alpha=0.05$ (two-sided) and $1-\beta=0.8$ (http://www.sample-size.net/), we needed 22 patients per group, or 44 patients. To allow for few dropouts, we decided to include 50 patients ( 25 in each group). When using these same reference values, a group comparison based on a paired $t$ test with a total of 22 patients has a statistical power of $97 \%$.

\section{Statistical analysis}

Unless otherwise specified, continuous variables are presented as mean (SD) and categorical variables as number and proportion. Nociception level parameters were not normally distributed; they are presented as median [interquartile range (IQR)] and were logtransformed prior to analysis.
To account for the crossover design, post-stimulation responses for the NOL index (primary endpoint), HR, and the AUC for the NOL index and HR (secondary endpoints) were analyzed using repeated measures analysis of variance (ANOVA) models accounting for group (MAC, GVL), period (laryngoscopy \#1, laryngoscopy \#2), and order of the two laryngoscopies (MAC first than GVL or GVL first than MAC) to test the carry-over effect and the group effect. In case of significant carry-over effect (alpha $=0.05)$, the two groups were compared using a standard Student $t$ test based on data from laryngoscopy \#1 only, otherwise data from both periods have been retained to produce group comparison.

As exploratory purposes, for laryngoscopies \#1 and \#2 as well as for laryngoscopy \#3 + tracheal intubation, pre and post-stimulation responses to clinical stimuli were analyzed using repeated measures ANOVA models with terms for time (pre and post-stimulation), group (MAC, GVL) and time $\times$ group interaction. Paired $t$ tests were also used to compare post-simulation responses and AUCs between laryngoscopy \#1 and laryngoscopy \#3 + intubation, and both groups combined.

Receiver operating characteristic (ROC) curves were constructed to evaluate the ability of the individual parameter to discriminate between noxious and nonnoxious states and confidence intervals of the AUCs were calculated. Youden's index was used to define the best cutoff value. $^{24}$

Statistical analyses were done using SAS version 9.4 and were performed at a two-sided 0.05 significance level. No adjustments were made for multiple comparisons.

\section{Results}

Demographic data are presented in Table 1. Data on NOL, $\mathrm{HR}$, and BIS before and after stimulations are presented in Table 2 for laryngoscopy \#1 and \#2, as well as for laryngoscopy \#3+ endotracheal intubation. Figure 3 presents the four parameters (HR, mean arterial pressure, NOL, and BIS) as mean values over time from $30 \mathrm{sec}$ before stimulation and for three minutes after.

Primary analysis

When analyzing post-stimulation NOL index combining both laryngoscopies \#1 and \#2 under the crossover design, no carry-over effect was detected from laryngoscopy \#1 to laryngoscopy \#2 $(P=0.56)$. The median [IQR] poststimulation NOL index peak was significantly lower for GVL than for MAC (14.8 [6.5-21.0] vs 18.5 [10.0-25.4], respectively; $P=0.01)$. 
Table 1 Demographic data of the patients. Continuous variables are presented as mean (SD) and categorical variables as $n(\%)$

\begin{tabular}{|c|c|}
\hline & Randomized patients $(n=49)$ \\
\hline Age, yr & $51.2(16.2)$ \\
\hline $18-29 \mathrm{yr}$ & $3(6.1 \%)$ \\
\hline 30-49 yr & $21(42.9 \%)$ \\
\hline $50-69 \mathrm{yr}$ & $17(34.7 \%)$ \\
\hline $70 \mathrm{yr}$ and more & $8(16.3 \%)$ \\
\hline \multicolumn{2}{|l|}{ Sex } \\
\hline Male & $11(22 \%)$ \\
\hline Female & $38(78 \%)$ \\
\hline BMI, $\mathrm{kg} \cdot \mathrm{m}^{-2}$ & $26.2(3.4)$ \\
\hline$<18$ & $2(4 \%)$ \\
\hline $18-24.9$ & $15(31 \%)$ \\
\hline $25-29.9$ & $24(49 \%)$ \\
\hline $30-35$ & $8(16 \%)$ \\
\hline \multicolumn{2}{|l|}{ ASA physical status } \\
\hline I & $19(39 \%)$ \\
\hline II & $30(61 \%)$ \\
\hline III & 0 \\
\hline \multicolumn{2}{|l|}{ Cormack-Lehane grade } \\
\hline 1 & $24(49 \%)$ \\
\hline 2 & $25(51 \%)$ \\
\hline \multicolumn{2}{|l|}{ Spectrum $^{\mathrm{TM}}$ LoPro blade size } \\
\hline \#3 & $38(78 \%)$ \\
\hline \#4 & $11(22 \%)$ \\
\hline \multicolumn{2}{|l|}{ Macintosh blade size } \\
\hline \#3 & $38(78 \%)$ \\
\hline \#4 & $11(22 \%)$ \\
\hline \multicolumn{2}{|l|}{ Endotracheal tube size } \\
\hline \#7 & $38(78 \%)$ \\
\hline \#8 & $11(22 \%)$ \\
\hline Mouth opening (cm) & $3.98(0.65)$ \\
\hline $3-3.9$ & $30(61 \%)$ \\
\hline $4-4.9$ & $14(29 \%)$ \\
\hline $5 \mathrm{~cm}$ and more & $5(10)$ \\
\hline Thyromental distance $(\mathrm{cm})$ & $7.35(0.83)$ \\
\hline$<6$ & 0 \\
\hline $6-6.9$ & $15(31 \%)$ \\
\hline $7-7.9$ & $20(41 \%)$ \\
\hline $8 \mathrm{~cm}$ and more & $14(28 \%)$ \\
\hline \multicolumn{2}{|l|}{ Comorbidities } \\
\hline Hypertension & $15(31 \%)$ \\
\hline Dyslipidemia & $9(18 \%)$ \\
\hline GERD & $6(12 \%)$ \\
\hline History of depression & $6(12 \%)$ \\
\hline Hypothyroidism & $5(10 \%)$ \\
\hline Moderate anemia & $4(8 \%)$ \\
\hline Chronic bronchitis & $3(8 \%)$ \\
\hline Diabetes & $2(4 \%)$ \\
\hline
\end{tabular}

Table 1 continued

\begin{tabular}{lc}
\hline & Randomized patients $(n=49)$ \\
\hline Smoker & $2(4 \%)$ \\
\hline
\end{tabular}

Date are mean (standard deviation) or percentage (\%) as indicated. ASA = American Society of Anesthesiologists physical status; $\mathrm{BMI}=$ body mass index; GERD = gastroesophageal reflux disease.

Secondary analysis

Post-stimulation HR and AUC of NOL index and HR were also analyzed using a crossover model and no carry-over effect was detected from laryngoscopy \#1 to laryngoscopy $\# 2$ ( $P=0.93,0.56$ and 0.89 , respectively).

The AUC value for the NOL index was significantly lower for GVL than for MAC (252.5 [125.5-446.5] vs 301.0 [195.5-443.0], respectively; $P=0.03$ ). No statistically significant differences were found between MAC and GVL for post-stimulation HR and AUC of HR (Table 2).

Deidentified data for NOL, HR, MAP, and BIS can be found in the Electronic Supplementary Material ([ESM]; eAppendix).

\section{Exploratory analysis}

Nociception level index values pre-laryngoscopy (baseline NOL values) were not significantly different between MAC and GVL groups and are presented in the ESM (eTable). As expected, laryngoscopy \#1 and \#2 created a noxious stimulus, which significantly increased NOL values between pre- and post-stimulation for both MAC and GVL techniques $(P<0.001$ for all laryngoscopies, see eTable, ESM).

Nociception level index values pre-laryngoscopy \#3 (baseline NOL values) were not statistically different between GVL and MAC groups $(P=0.49)$. Not surprisingly, NOL values after laryngoscopy \#3+ intubation (either with MAC or GVL blade) were significantly higher than the NOL values after laryngoscopies \#1 and \#2 combined without intubation Table 2). Nociception level values after laryngocopy \#3+ intubation were not significantly different between MAC and GVL groups $(P=0.50$; Table 2$)$.

Increases in delta NOL values after laryngoscopy \#3+ intubation were statistically significant between the devices $(P<0.001)$ but did not show any significant difference between MAC and GVL groups $(P=0.66)$. Also, the AUC of NOL values from $\mathrm{T} 0$ until $\mathrm{T}+3$ min after laryngoscopy $\# 3+$ intubation were not significantly different between 
Table 2 Post-stimulation values for nociception level index and heart rate after airway stimulation with direct or videolaryngocopy \pm endotracheal tube insertion

\begin{tabular}{|c|c|c|c|c|}
\hline Parameter & Type of stimuli & GVL & MAC & $P$ value \\
\hline \multirow[t]{4}{*}{ Mean peak NOL } & Laryngo 1 (L1) & $9.4[5.5-18.1]$ & $15.8[8.7-23.4]$ & Test for carry-over effect: 0.56 \\
\hline & Laryngo 2 (L2) & $18.7[12.1-29.5]$ & $19.1[10.9-27.0]$ & \\
\hline & Combined laryngo 1 (L1) and laryngo 2 (L2) & $14.8[6.6-21.0]$ & $18.5[10.0-25.4]$ & 0.01 \\
\hline & Laryngo 3 & $55.7[36.6-67.9]$ & $51.57[35.5-58.4]$ & 0.50 \\
\hline \multirow[t]{4}{*}{ Mean peak HR } & Laryngo 1 (L1) & $68.2(11.1)$ & $68.4(11.7)$ & Test for carry-over effect: 0.93 \\
\hline & Laryngo 2 (L2) & $68.3(11.6)$ & $69.10(10.5)$ & \\
\hline & Combined laryngo 1 (L1) and laryngo 2 (L2) & $68.3(11.2)$ & $68.7(10.0)$ & 0.27 \\
\hline & Laryngo 3 & $84.5(14.4)$ & $80.4(12.2)$ & 0.29 \\
\hline \multirow[t]{4}{*}{ AUC NOL } & Laryngo 1 (L1) & $151.8[106.0-325.5]$ & $296.0[195.5-455.0]$ & Test for carry-over effect: 0.56 \\
\hline & Laryngo 2 (L2) & $361.5[247.0-598.5]$ & $304.0[194.8-424.8]$ & \\
\hline & Combined laryngo 1 (L1) and laryngo 2 (L2) & $252.5[125.5-446.5]$ & $301.0[195.5-443.0]$ & 0.03 \\
\hline & Laryngo 3 & $\begin{array}{l}1386.5 \\
{[832.5-1630.2]}\end{array}$ & $\begin{array}{l}1133.0 \\
{[807.0-1570.0]}\end{array}$ & 0.70 \\
\hline \multirow[t]{4}{*}{ AUC HR } & Laryngo 1 (L1) & $2385.3(383.5)$ & $2383.5(392.9)$ & Test for carry-over effect: 0.89 \\
\hline & Laryngo 2 (L2) & $2387.3(397.8)$ & $2416.4(357.5)$ & \\
\hline & Combined laryngo 1 (L1) and laryngo 2 (L2) & $2386.3(386.8)$ & $2399.62(372.4)$ & 0.36 \\
\hline & Laryngo 3 & $2806.4(426.2)$ & $2672.6(385.6)$ & 0.25 \\
\hline
\end{tabular}

$\mathrm{GVL}=$ GlideScope $^{\mathrm{TM}}$ videolaryngoscopy using Spectrum ${ }^{\mathrm{TM}}$ angulated LoPro Single-Use Blade. MAC = direct laryngoscopy using reusable Macintosh blade. $\mathrm{HR}=$ heart rate; NOL = nociception level; laryngo 1 or L1 = laryngoscopy \#1; laryngo 2 or L2 = laryngoscopy \#2; laryngo 3 or L3 = laryngoscopy \#3; Data are expressed, based on their distribution, as median [interquartile range] for the NOL index and mean (standard deviation) for HR. AUC $=$ area under the curve for NOL and HR after stimulation from time T0 (stimulus applied) and T $+180 \mathrm{sec}$ after stimulus. The $P$ value at the end of each row is associated with the comparison between GVL and MAC. Mean peak value stands for the fact that the peak of each study parameter was an average of five values around the actual single value peak for the parameter to avoid artefacts being considered as peak (see Methods).

MAC and GVL groups (AUC 1133.0 [807.0-1570.0] vs 1386.50 [832.5-1630.3], respectively; $P=0.70$; Table 2 and Fig. 3).

When comparing NOL for laryngoscopy \#1 and 2 combined (no tracheal intubation) vs laryngoscopy \#3 (with tracheal intubation), post-stimulus NOL values were significantly higher for laryngoscopy \#3 + intubation than for laryngoscopy \#1 + $2(52.5$ [35.5-63.0] vs 14.8 [6.5-21.0], $P<0.001$; Fig. 4 and Table 2), delta NOL values post-laryngoscopy $\# 3+$ intubation were significantly higher than delta NOL values postlaryngoscopy \#1 $(P<0.001)$ and the AUC of NOL values from T0 until $\mathrm{T}+3 \mathrm{~min}$ after laryngoscopy \#3+ intubation were also significantly higher than those calculated for laryngoscopies \#1 + 2 (AUC 1,268.0 [807.0-1,593.5] vs 252.5 [125.5-446.5] respectively; $P<$ 0.001; Fig. 3 and Table 2).

\section{Secondary outcomes}

As for the NOL index, standard hemodynamic parameters were significantly increased pre- $v s$ post-stimulation for laryngoscopy \#1, \#2, and \#3 (Table 2 and ESM eTable).
For HR, baseline values did not significantly differ between MAC and GVL groups at laryngoscopy \#1 +2 and \#3 (ESM, eTable). As mentioned above, post-stimulation HR values were significantly higher after stimulation at laryngoscopy \#1 + 2 vs laryngoscopy \#3 + intubation (Table 2). Post-stimulation HR values were not different between MAC and GVL groups at laryngoscopy \#1 +2 and $\# 3+$ intubation $(P=0.267$ and $P=0.288$, respectively). Also, AUC of HR values did not show any significant difference between MAC and GVL at laryngoscopy \#1 +2 or laryngoscopy \#3 + intubation (Fig. 3, Table 2, and ESM eTable). Also, comparison of delta HR values between MAC and GVL groups did not show a significant difference, no matter which laryngoscopy was used (Fig. 4). This suggests HR did not discriminate between MAC and GVL for laryngoscopies \#1 +2 or laryngoscopy \#3 + intubation.

For the BIS index, post-stimulation values were all significantly higher than the pre-stimulation values (ESM, eTable). Nevertheless, post-stimulation BIS did not discriminate between MAC and GVL at laryngoscopy \#1 +2 (ESM, eTable) and at laryngoscopy \#3 + intubation $(P=0.543)$. The MAP values are displayed in Fig. 3 . We 

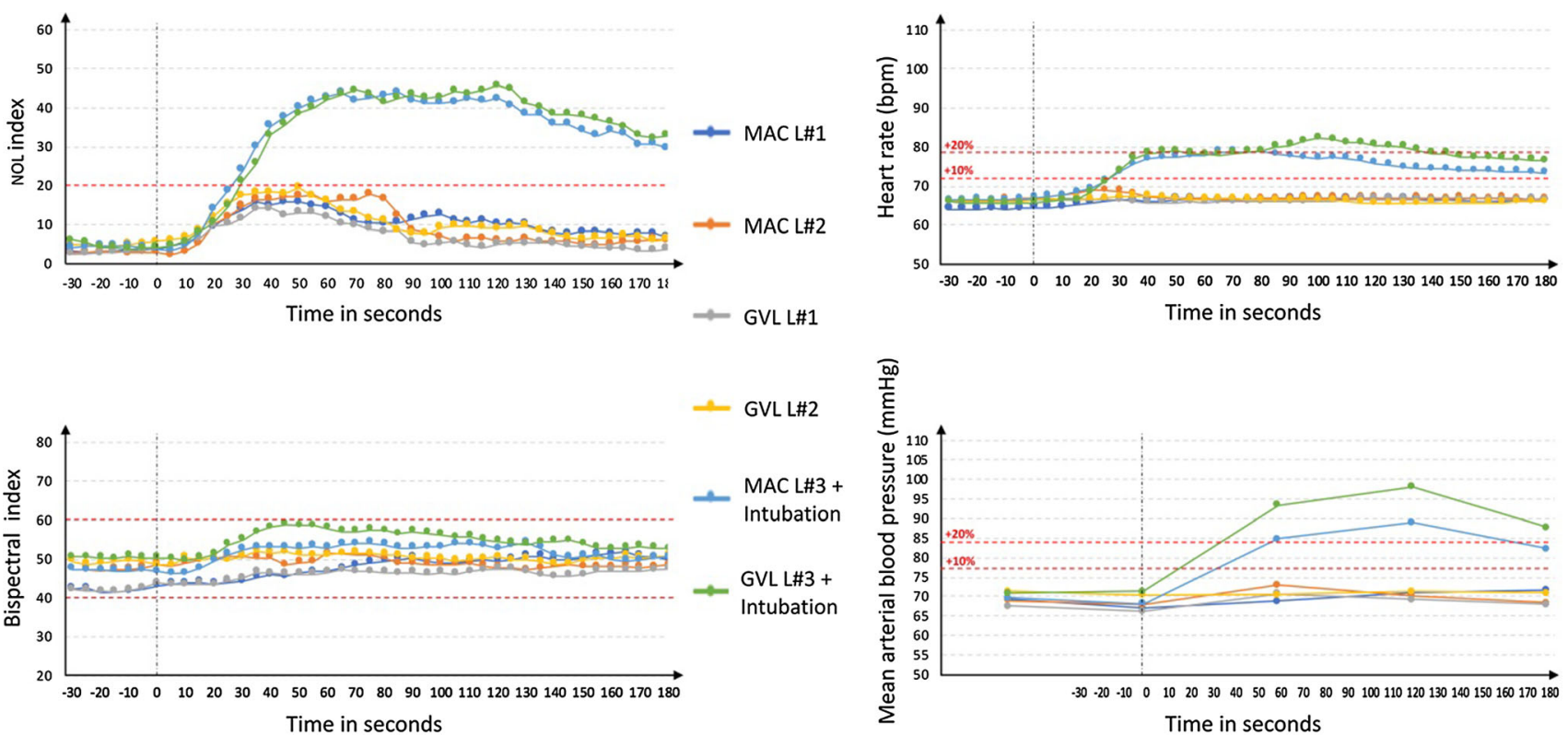

Fig. 3 Representation of all four study parameters (NOL index, HR, BIS, mean arterial blood pressure) from $30 \mathrm{sec}$ prior to the stimulation (stimulation occurs a T0, vertical black dashed line) and for three minutes after stimulation. NOL, HR and BIS were recorded and are presented every five seconds. As non-invasive blood pressure measurement was taken only every minute (no arterial line necessary in these patients) for the duration of the study, mean arterial blood pressure is presented every minute. For the NOL index the red horizontal dashed line set at $\mathrm{NOL}=20$ represents the nociceptive threshold classically reported in previous studies as clinically meaningful and above which clinicians might treat

did not think it necessary to present all the data and statistical analysis for MAP values, which were recorded every minute. Finally, ROC curves were built (as previously reported ${ }^{19}$ ) for the laryngoscopy \#3 + intubation and showed that the NOL index had netter sensitivity and specificity than HR and BIS index or MAP (Fig. 5).

\section{Discussion}

This randomized clinical trial prospectively evaluated and compared the influence of direct and video laryngoscopy with or without tracheal intubation on the nociceptive response induced by this type of stimulation as assessed by a nociception index. Previous studies only evaluated the changes in more classical hemodynamic parameters (HR and blood pressure) during laryngoscopy and intubation with video and direct laryngoscopy. The present study adds an evaluation with a more sensitive and specific monitor to characterize nociceptive response, the NOL index from the PMD200 ${ }^{\mathrm{TM}}$ device. ${ }^{16-19}$ The automated collection of NOL values, as well as HR and BIS every five seconds, allowed nociception if NOL index stays above this value for more than one or two minutes. For heart rate $\left(\mathrm{bpm}=\right.$ beats $\left.\cdot \mathrm{min}^{-1}\right)$ and for mean arterial blood pressure, the red horizontal dashed lines represent + $10 \%$ of variation or $+20 \%$ of variation from the baseline values of the parameter (average of the pre-stimulation values for the $30 \mathrm{sec}$ preceding the stimulus). For BIS, the red horizontal dashed lines represent the classically described values (40-60) for BIS clinical use. Values presented here are means for all parameters at each timepoint. $\mathrm{BIS}=$ bispectral index; $\mathrm{HR}=$ heart rate; $\mathrm{NOL}=$ nociception level.

us to analyze the parameters more precisely and created rigorous and more precise data records and analyses.

The present study analyzed the nociceptive response to laryngoscopy alone (without performing the classical consequent tracheal intubation) and showed that videolaryngoscopy induced significantly less nociceptive response than direct laryngoscopy, meaning the nociceptive response measured by the NOL index was less intense $(P=0.01$ for laryngoscopies 1 and 2 combined). The AUC value for the NOL index after laryngoscopy \#1 was also lower with the GVL technique than with MAC technique $(P=0.023)$. This showed that when using either post-stimulation average of peak values or a post-stimulation AUC calculation to compare GVL with MAC, GVL induces significantly less nociception measured by the NOL index than MAC when only laryngoscopy without tracheal intubation is performed under standardized general anesthesia. Nevertheless, performing laryngoscopy without intubation is very rare in anesthesia practice. These findings on laryngoscopies with GVL vs MAC mainly serve to better the difference between the two techniques. The impact of laryngoscopy + intubation further explored in this study with laryngoscopy \#3 is remains the most clinically relevant finding. 


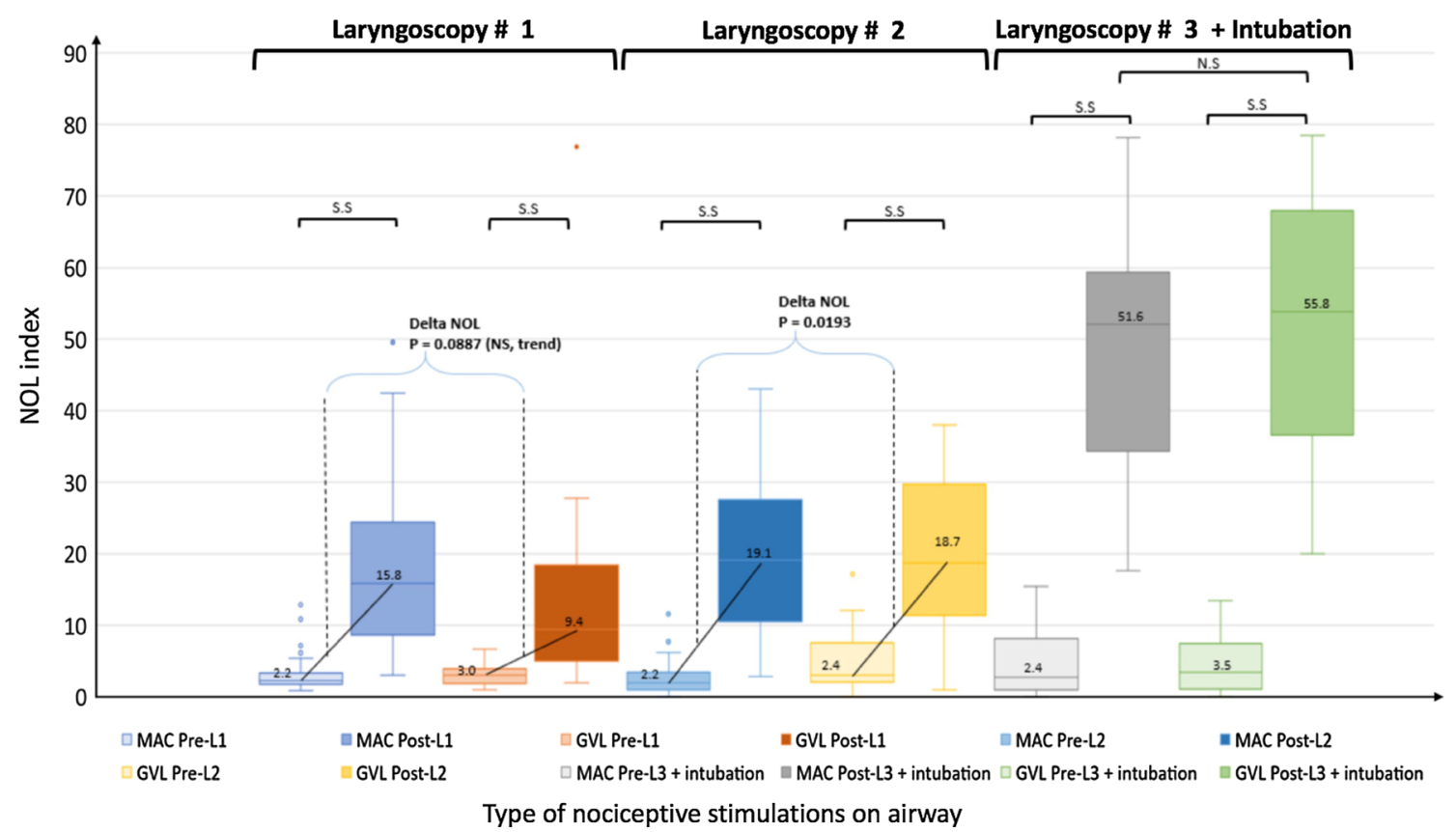

Fig. 4 NOL index variations after six different stimulations: laryngoscopy \# 1 with MAC or GVL, laryngoscopy \#2 with MAC or GVL, laryngoscopy \#3 + intubation with MAC or GVL. Pre(baseline) and post-stimulation values are presented for each stimulation. NOL values are presented as median [interquartile range]. Delta NOL $=$ absolute difference between pre- $v s$ poststimulation values; GVL $=$ GlideScope ${ }^{\mathrm{TM}}$ videolaryngoscopy with

In comparison with the NOL index, HR, a more classically used clinical parameter to assess intraoperative nociception, was able to detect nociception induced by all types of laryngoscopy in this study when comparing pre- $v s$ post-stimulation values (Table 2 and ESM eTable). Nevertheless, post-stimulation HR values were not able to differentiate between MAC and GVL when laryngoscopy \#1 and \#2 were combined $(P=0.27)$. Also, the AUCs of HR values after laryngoscopy \#1 and \#2 were not significantly different between MAC and GVL $(P=$ 0.36). This emphasizes that HR, as previously reported, ${ }^{15-19}$ is less sensitive than the NOL index at detecting nociceptive stimulation under general anesthesia. This was also reinforced by the ROC curve analysis, which showed that for laryngoscopy \#3 + intubation, the NOL index is more sensitive than the HR (Fig. 5).

Not surprisingly, the present study showed that average pre- $v s$ post-stimulation values for the NOL index, HR, $\mathrm{BIS}$, and MAP were able to detect nociceptive stimulation when laryngoscopy \#1, \#2, and \#3 + intubation were performed either with MAC or GVL (Table 2 and ESM eTable). The question is what are anesthesiologists supposed to do with a parameter that increases by less than $10 \%$ of the baseline values for HR and MAP (Fig. 3)? Even if statistically significant, will the anesthesiologist be able to perceive it by eye, and will it change anesthesia
Spectrum $^{\mathrm{TM}}$ LoPro Single-Use blade; L1 = laryngoscopy \#1; L2 = laryngoscopy \#2; L3 = laryngoscopy \#3; MAC = direct laryngoscopy withMacIntosh blade; $\mathrm{NOL}=$ nociception level; N.S. $=$ not significant; S.S. = statistically significant.

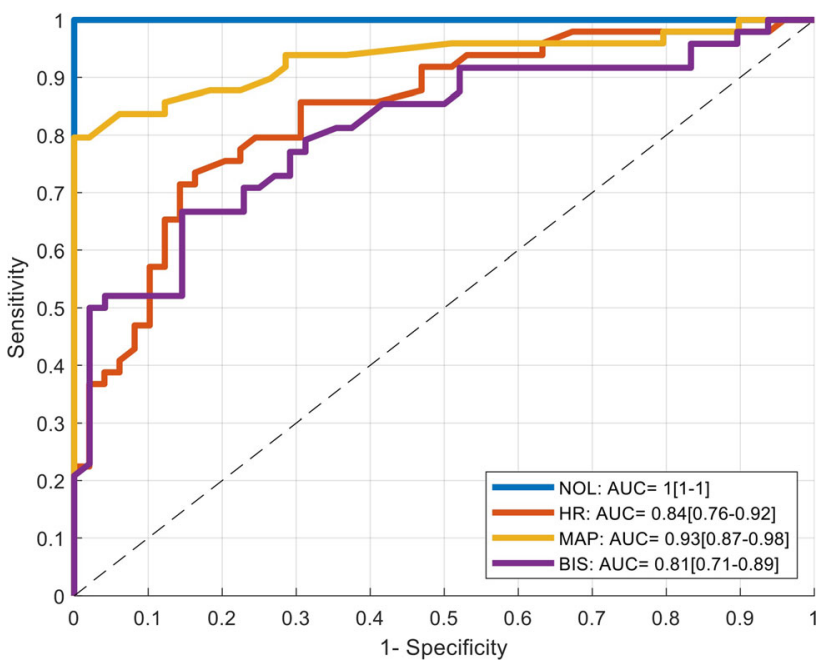

Fig. 5 Receiver operating characteristics (ROC) curve analysis of discrimination of experimental noxious stimulus. Analysis done for laryngoscopy \#3 + intubation at remifentanil dosage $0.05 \mu \mathrm{g} \mathrm{kg}^{-}$ ${ }^{1} \mathrm{~min}^{-1}$ and propofol $100 \mu \mathrm{g} \mathrm{kg}^{-1} \mathrm{~min}^{-1}$. AUC $=$ area under the receiver operating characteristic curve; $\mathrm{BIS}=$ bispectral index; $\mathrm{HR}=$ heart rate; $\mathrm{MABP}=$ mean arterial pressure; $\mathrm{NOL}=$ nociception level.

practice? Anesthesia clinicians will need to determine the difference between a statistically significant difference and the clinical relevance of this statistically significant difference. A similar point might be raised for the BIS in 
our results, which remained within the range of 40-60 after most stimulations, so was not helpful in terms of clinical decision-making even if pre- $v s$ post-stimulations values were significantly different (Fig. 3, Table 2). The analysis of sensitivity and specificity of each index showed that the NOL index had better sensitivity at the same specificity than HR, BIS, and MAP. Also, because the NOL index offers a nociceptive threshold previously reported to be around the NOL value of 20 on a scale of 100, this might make this index easier to use in clinical practice to guide the administration of intraoperative medication to alleviate nociception.

Here, the NOL index was the only parameter able to differentiate nociception induced by laryngoscopy alone (without tracheal intubation) when comparing the GVL vs the MAC techniques $(P=0.01)$.

A secondary objective of this study was to evaluate and properly quantify whether there was a difference in terms of post-stimulation induced nociception between laryngoscopy alone (with MAC or GVL and without any tracheal intubation) and laryngoscopy + subsequent tracheal intubation. Laryngoscopy with endotracheal tube insertion between the vocal cords and into the trachea (laryngoscopy \#3 + tube) induced a significantly higher nociceptive response than laryngoscopy alone (without intubation, laryngoscopy \# 1 and \#2) with both blades combined. This was shown by the significant differences in the post-stimulation median [IQR] NOL index values (13.0 [8.1-20.0] for laryngoscopy \#1 and 52.6 [35.6-63.0] for laryngoscopy \#3 + tube; $P<0.001)$ and in the poststimulation mean (SD) HR values [68.3 (11.3) vs 82.5 (13.4), respectively; $P<0.001]$. This is the first time that such a difference in nociception induced by laryngoscopy alone vs laryngoscopy with intubation has been evaluated and showed significantly more "painful" for the laryngoscopy + intubation procedure. This is not surprising; however, it is of major interest to better design future studies that will assess peri-intubation effect of medication or strategies. Also, this difference in nociception induced by laryngoscopy alone and laryngoscopy + intubation shown in our study might provide a useful basis for a future study that will assess the use of supra- and sub-glottic lidocaine spray to decrease and perhaps avoid the nociceptive reaction we observed when the tube was inserted into the trachea. Heart rate and NOL index might be helpful parameters in such a study to diagnose nociception induced by the procedure.

The limitations of the present study include the fact that the anesthesiologist who performed the procedures was not blinded to the device that was used, as this was impossible. Nevertheless, as all the criteria were electronically recorded every five seconds, there is no chance that the operator might have influenced them. Also, the fact that three stimulations were performed on each study patient might be seen as a limitation as one could argue that repeated stimulations of the airway might have influenced the nociceptive response from laryngoscopy \#1+ 2 (crossover part of the study which showed no carry-over effect between L1 and L2) to laryngoscopy \#3, with higher expected response at laryngoscopy \#3. We recognize this might be a limitation and this is why we designed this study as a crossover study with two randomizations. The statistical analysis made to evaluate whether there was any carry-over effect from the first laryngoscopy on the second one showed, for HR and the NOL index, that there was no carry-over effect, which means that results following the second laryngoscopy stimulation could be considered free from bias due to the first laryngoscopy. Certainly, this might be because we allowed for sufficient time between two stimulations to completely recover from the stimulation (see Fig. 3), and showed that HR and the NOL index returned to normal pre-stimulation values within the three minutes of the laryngoscopy \#1 and \#2 stimulations.

The selection of the best view (either grade 1 or 2 Cormack-Lehane view) to complete the laryngoscopies (laryngoscopy 1 and 2), then the laryngoscopy + intubation (laryngoscopy 3) might be seen as a weakness of the study design as a Cormack-Lehane grade 1 should have been sought for all patients to actually assess the difference in noxious force to achieve a defined view between MAC and GVL. Nevertheless, the results already showed a significant difference in the NOL index variations following laryngoscopies with MAC and GVL techniques. This difference might have been even stronger if we had chosen to obtain values for all patients with Cormack-Lehane grade 1 . Also, $51 \%$ of our study had a Cormack-Lehane grade of 2 at the laryngoscopy with MAC, and it is unclear if we would have achieved grade 1 by applying more force during the laryngoscopy.

Also, one could argue that stimulation \#3 was performed much further from the initial induction of anesthesia than stimulation \#1, which could mean that remifentanil and propofol effects would be higher at stimulation \#1 than at \#2 and \#3. Remifentanil was set at a rate of $0.05 \mathrm{mcg} \cdot \mathrm{kg}^{-}$ ${ }^{1} \cdot \mathrm{min}^{-1}$ and propofol at $100 \mu \mathrm{g} \cdot \mathrm{kg}^{-1} \cdot \mathrm{min}^{-1}$ at the end of the induction boluses. Laryngoscopies \#1, \#2, and \#3 were performed at T5, T9, and T13 after the boluses and were under the same infusion for both drugs. After an intravenous bolus, remifentanil plasma concentration peaks around 60-90 sec and then start to decrease. At T5 minutes after the end of the bolus and under remifentanil infusion of $0.05 \mu \mathrm{g} \cdot \mathrm{kg}^{-1} \cdot \mathrm{min}^{-1}$, the plasma concentration of remifentanil might be considered almost identical at $\mathrm{T} 5$, T9, and T13. This is also emphasized by the results of the study after the same type of stimulation at laryngoscopy \#1 
and \#2: the peak of HR and the peak of NOL were not statistically different for the same stimulation.

Finally, our study used the NOL index, recently validated for clinical use in anesthetized patients by Health Canada in September 2017. This means that, currently, very few centres in Canada use this index, which might limit the usefulness of this study. Nevertheless, we also combined the evaluation of the NOL index with the more classical parameters HR, blood pressure, and BIS to show that the NOL index is the same if not better than these classic parameters. This study will invigorate the discussion of whether anesthesiologists should use better indexes to assess intraoperative nociception than classical parameters such as HR.

\section{Conclusion}

The NOL index helped show that the nociception induced by laryngoscopy alone (without tracheal intubation) was significantly lower with the GlideScope ${ }^{\mathrm{TM}}$ Titanium Spectrum LoPro Single-Use blades than direct laryngoscopy with reusable Macintosh blades. Nevertheless, performing a laryngoscopy alone in anesthesia practice is rare; thus, this observation has limited clinical relevance. Nevertheless, when both laryngoscopy + tracheal intubation were performed, the nociceptive response was significantly greater than with laryngoscopy alone, though no difference between the type of blade used was observed. Finally, the NOL index showed better ability to detect nociception induced by laryngoscopy and laryngoscopy + intubation than the more classical parameters, such as HR, BIS, and MAP. Further studies will have to evaluate the impact of different strategies to reduce the impact of the tracheal intubation to improve the clinical benefits that can be observed from laryngoscopy.

\begin{abstract}
Author contributions Virginie Sbeghen, Olivier Verdonck, Valerie Zaphiratos, Véronique Brulotte, Christian Loubert, Issam Tanoubi, Marie-Eve Belanger, Nadia Godin, Marie-Claude Guertin, and Annik Fortier contributed to design, execution, analysis, writing, revision, and agree with the content of the article. Jason McDevitt contributed to analysis, revision, and agrees with the content of the article. Pierre Drolet contributed to design, analysis, revision, and agrees with the content of the article. Louis-Philippe Fortier contributed to design, revision, and agrees with the content of the article. Philippe Richebe was the principal investigator of the study and contributed to the design, administrative agreements (scientific, ethic, institution), execution, analysis, interpretation, drafting, writing, revision, and agrees with the content of the article and final approval and submission.
\end{abstract}

Acknowledgements The PMD200 ${ }^{\mathrm{TM}}$ device was a loan from Medasense LTD that also offered the supplies for the NOL index evaluation for this study. Thanks to Moulay Idrissi who was the research assistant for this study. Thanks to Kyle Vaughn Roerick, editor, for his English language edition of this manuscript. This study was awarded the third prize at the resident competition of the Association of Anesthesiologists of the province of Quebec in 2019 and was also presented at the American Society of Anesthesiology meeting as an e-poster in 2018, San Francisco, CA, USA.

Disclosures Dr. Philippe Richebé was a member of the advisory board of the company Medasense until 2020 and a consultant for this company. Nevertheless, all the studies he performed as a PI were done after a contract signed between both the parties (attorneys of the Research Center of HMR-CEMTL and the companies) and always via an Independent Investigator Initiated Trial grant-contract. He also received honoraria as medical consultant from Abbvie, Medtronic, Biosyent, Edwards, Merck, and Avirpharma for lectures.

There is no conflict of interest between the present study/article and the above cited contracts.

Funding statement This study was partly supported by an Independent Investigator Initiated Trial Research Grant from Verathon, Bothell, WA, USA as well as CHS, Oakville, ON, Canada, and by the Department of Anesthesiology of MaisonneuveRosemont Hospital.

Editorial responsibility This submission was handled by $\mathrm{Dr}$. Gregory L. Bryson, former Deputy Editor-in-Chief, Canadian Journal of Anesthesia.

\section{References}

1. Choi HJ, Kim YM, Oh YM, et al. GlideScope video laryngoscopy versus direct laryngoscopy in the emergency department: a propensity score-matched analysis. BMJ Open 2015. https://doi. org/10.1136/bmjopen-2015-007884.

2. Griesdale DE, Liu D, McKinney J, Choi PT. Glidescope ${ }^{\circledR}$ videolaryngoscopy versus direct laryngoscopy for endotracheal intubation: a systematic review and meta-analysis. Can J Anesth 2012; 59: 41-52.

3. Ibinson JW, Ezaru CS, Cormican DS, Mangione MP. GlideScope use improves intubation success rates: an observational study using propensity score matching. BMC Anesthesiol 2014. https:// doi.org/10.1186/1471-2253-14-101.

4. Misirlioglu G, Sen $O$. Comparison of GlideScope video laryngoscopy and Macintosh laryngoscope in ear-nose and throat surgery. Ir J Med Sci 2016; 185: 729-33.

5. Savoldelli GL, Schiffer E, Abegg C, Baeriswyl V, Clergue F, Waeber JL. Comparison of the Glidescope, the McGrath, the Airtraq and the Macintosh laryngoscopes in simulated difficult airways. Anaesthesia 2008; 63: 1358-64.

6. Caldiroli D, Molteni F, Sommariva A, et al. Upper limb muscular activity and perceived workload during laryngoscopy: comparison of Glidescope $\AA$ and Macintosh laryngoscopy in manikin: an observational study. Br J Anaesth 2014; 112: 563-9.

7. Carassiti $M$, Biselli V, Cecchini $S$, et al. Force and pressure distribution using Macintosh and GlideScope laryngoscopes in normal airway: an in vivo study. Minerva Anestesiol 2013; 79: 515-24.

8. Lee C, Russell T, Firat M, Cooper RM. Forces generated by Macintosh and GlideScope ${ }^{\circledR}$ laryngoscopes in four airwaytraining manikins. Anaesthesia 2013; 68: 492-6. 
9. Lee RA, van Zundert AA, Maassen RL, Wieringa PA. Forces applied to the maxillary incisors by video laryngoscopes and the Macintosh laryngoscope. Acta Anaesthesiol Scand 2012; 56: 224-9.

10. Mitsdarffer B, Browder M. Comparison of the force required to perform endotracheal intubation on healthy adult patients utilizing the GlideScope ${ }^{\circledR}$ videolaryngoscope and Macintosh laryngoscope: a systematic review protocol. JBI Database System Rev Implement Rep 2015; 13: 35-44.

11. Russell T, Lee C, Firat M, Cooper RM. A comparison of the forces applied to a manikin during laryngoscopy with the GlideScope and Macintosh laryngoscopes. Anaesth Intensive Care 2011; 39: 1098-102.

12. Siddiqui N, Katznelson R, Friedman Z. Heart rate/blood pressure response and airway morbidity following tracheal intubation with direct laryngoscopy, GlideScope and Trachlight: a randomized control trial. Eur J Anaesthesiol 2009; 26: 740-5.

13. Xue FS, Zhang GH, Li XY, et al. Comparison of hemodynamic responses to orotracheal intubation with the GlideScope videolaryngoscope and the Macintosh direct laryngoscope. J Clin Anesth 2007; 19: 245-50.

14. Benjamin FJ, Boon D, French RA. An evaluation of the GlideScope, a new video laryngoscope for difficult airways: a manikin study. Eur J Anaesthesiol 2006; 23: 517-21.

15. Ben-Israel $N$, Kliger $M$, Zuckerman $G$, Katz $Y$, Edry $R$. Monitoring the nociception level: a multi-parameter approach. J Clin Monit Comput 2013; 27: 659-68.

16. Edry R, Recea V, Dikust Y, Sessler DI. Preliminary intraoperative validation of the nociception level index: a noninvasive nociception monitor. Anesthesiology 2016; 125: 193-203.

17. Martini CH, Boon M, Broens SJ, et al. Ability of the nociception level, a multiparameter composite of autonomic signals, to detect noxious stimuli during propofol-remifentanil anesthesia. Anesthesiology 2015; 123: 524-34.

18. Stockle PA, Julien M, Issa R, et al. Validation of the PMD100 and its NOL index to detect nociception at different infusion regimen of remifentanil in patients under general anesthesia. Minerva Anestesiol 2018; 84: 1160-8.

19. Renaud-Roy E, Stockle PA, Maximos S, et al. Correlation between incremental remifentanil doses and the Nociception Level (NOL) index response after intraoperative noxious stimuli. Can J Anesth 2019; 66: 1049-61.

20. Kohn JB. Adjusted or ideal body weight for nutrition assessment? J Acad Nutr Diet 2015. https://doi.org/10.1016/j.jand.2015.02. 007.

21. Krenitsky J. Adjusted body weight, pro: evidence to support the use of adjusted body weight in calculating calorie requirements. Nutr Clin Pract 2005; 20: 468-73.

22. Schwartz SN, Pazin GJ, Lyon JA, Ho M, Pasculle AW. A controlled investigation of the pharmacokinetics of gentamicin and tobramycin in obese subjects. J Infect Dis 1978; 138: 499-505.

23. Smetana KS, Ziemba R, May CC, Erdman MJ, Van Matre ET, Jones GM. Adjusted versus actual body weight dosing of 4-factor prothrombin complex concentrate in obese patients with warfarin-associated major bleeding. J Thromb Thrombolysis 2018; 47: 369-74.

24. Youden WJ. Index for rating diagnostic tests. Cancer 1950; 3 : 32-5.

Publisher's Note Springer Nature remains neutral with regard to jurisdictional claims in published maps and institutional affiliations. 